\title{
The ID Migraine in Migraine Headache Diagnosis: An Evaluation of its Usefulness in Calabar, Southern Nigeria
}

Oparah, Sidney K ${ }^{1 *}$, Asibong, Udeme $\mathrm{E}^{2}$

${ }^{1}$ Department of Internal Medicine, University of Calabar, Nigeria

${ }^{2}$ Department of Family Medicine, University of Calabar, Nigeria

DOI: 1 10.36348/SJMPS.2019.v05i10.010

| Received: $09.05 .2019 \mid$ Accepted: $22.05 .2019 \mid$ Published: 29.10 .2019

*Corresponding author: Oparah Sidney K

\section{Abstract}

Background: The under recognition and consequent mistreatment of migraine headache highlight the importance of quicker and easy to apply tools for migraine screening and diagnosis. The ID migraine fits the description of such a rapid screening diagnostic tool. Objective: To evaluate the ID migraine, in migraine headache diagnosis, among clinical students in Calabar, Nigeria. Methods: Using a cross sectional design, we compared the performance of the ID migraine with that of the IHS criteria, used as a gold standard for migraine headache diagnosis, on a set of persons with recurrent headaches. Participants were recruited from a pool of 220 apparently healthy clinical students of the University of Calabar. Data analysis was done with SPSS version 20; and the level of significance was set as $\mathrm{p}<0.05$. Results: Fifty one persons who had recurrent headaches from the pool of students, comprising $25(49 \%)$ males and $26(51 \%)$ females, proceeded to complete the study. The mean age of the participants was 24.22 years \pm 4.575 . The sensitivity, specificity, accuracy, positive predictive and negative predictive values we obtained for the ID migraine tool were $69.2 \%, 63.1 \%$, $64.7 \%, 39.1 \%$ and $85.7 \%$, respectively (kappa $=0.258 ; \mathrm{p}=0.043$ ). Conclusion: The ID migraine had moderate sensitivity and specificity in our locality, and a low level of agreement with the IHS criteria. Its usefulness in our locality may be more in ruling out, rather than ruling in, migraine in persons with recurrent headaches.

Keywords: ID migraine, headache, migraine diagnosis.

Copyright @ 2019: This is an open-access article distributed under the terms of the Creative Commons Attribution license which permits unrestricted use, distribution, and reproduction in any medium for non-commercial use (NonCommercial, or CC-BY-NC) provided the original author and source are credited.

\section{INTRODUCTION}

Migraine is a common type of primary headache which manifests with recurrent episodes of one sided, moderate to severe throbbing headaches lasting for 4 to 72 hours. The headaches are usually exacerbated by physical activity and accompanied by nausea, vomiting, aversion to light and sounds [1-6].

Migraine often starts around the time of puberty and affects the female sex more than males, with reported prevalence values for females reaching up to two to threefold higher than for males. The variations are attributed to hormonal influences [7-10. The interplay of environmental and genetic factors are believed to play significant contributions in the aetiology of migraine headaches in which activation of certain processes in the brain results to the elaboration of pain producing pro-inflammatory substances which act on intracranial blood vessels and nerves resulting in peripheral and central sensitization, with involvement of the trigemino-vascular system in the conveyance of nociceptive information $[10,11-14]$.
Globally, migraine is ranked among the top 20 causes of years of healthy life lost to disability [15]. In Africa, migraine is the $13^{\text {th }}$ leading cause of years lived with disability (YLD) in 2010 [16]. By 2030, Africa will have a projected $10 \%$ increase in migraine burden [17].

In response to the challenges posed by the wide reaching impact of headache disorders, the World Health Organization (WHO) and International Headache organizations launched the 'Lifting The Burden' initiative, which is a global campaign aimed at mitigating the global headache burden $[18,19]$. The campaign seeks to promote the vision of a world in which headache disorders are acknowledged as real, disabling and warranting medical care which is readily accessible irrespective of location. ${ }^{19}$

To enhance the goals of the global "Lifting the Burden" campaign against headaches, there is need for prompt recognition and accurate diagnoses of headache disorders to minimize mismanagement and wastage of 
limited available health resources. Limited emphasis and, in some cases, omission of diagnosis and management of headache disorders in the training of health-care providers give rise to health workers who are inexperienced in the management of headache disorders and therefore hesitant to offer health care in this field [19].

Despite the widely acknowledged problems associated with migraine headaches, the condition remains largely undertreated and underdiagnosed [20]. Diagnostic criteria and screening tools have been devised to ease and standardize the diagnoses of headache disorders [21]. The international headache Society (IHS) / ICHD-3 beta criteria for migraine headaches may prove to be cumbersome for nonspecialist healthcare professionals; especially in settings where patients heavily outnumber available clinicians. This highlights the importance of quicker and easy to apply tools for migraine screening and diagnosis [2225]. The ID migraine questionnaire fits the description of an easy to apply, time saving instrument employed in the evaluation of headaches for migraine $[23,26]$. The ID migraine questionnaire is a three item tool reported to have sensitivity, specificity and positive predictive values of $81 \%, 75 \%$ and $93 \%$, respectively [23].

Various categories of healthcare workers can be easily trained to become proficient in the use of the ID migraine questionnaire. Furthermore, the benefits of such an easily deployed assessment tools, to actualizing the goals of the campaign on lifting the burden of migraine, can hardly be overestimated in resource poor regions such as obtained in sub-Sahara Africa, grappling with the challenges posed by abysmal doctor: patient ratios [27]. However, variations in characteristics with varying cultural and geographic localities make validation of the ID migraine questionnaire in a given locality important for determining its usefulness in such given and similar populations.

In this study, we evaluated the applicability of the ID migraine questionnaire in a south-eastern Nigeria, Niger delta setting by comparing the tool with the International Headache Society (IHS) criteria for migraine diagnosis, among a population of undergraduate clinical students at the University of Calabar, Nigeria.

\section{METHODS}

This study was conducted in Calabar, the capital city of Cross river state, in southern Nigeria. The city located at latitude $4^{\circ} 58^{\prime \prime} \mathrm{N}$ and longitude $8^{\circ} 17^{\prime} \mathrm{E}$, is a major tourist destination in Nigeria, with a population of 371,122 , comprising of 186,607 males and 184,415 females, during the last national population census [28].
The study was conducted after obtaining approval and the process was carried out in agreement with the Helsinki declaration of 1975, as revised in 1983. In this cross-sectional design study, we employed a multi stage sampling technique with the participants drawn from undergraduate clinical students of the University of Calabar, Nigeria.

A pool of the medical students, of the aforementioned university, who were at the clinical class levels were initially screened for history of headache within the previous three months. Those who reported experiencing headache were further screened for recurrent headaches; defined as having at least two episodes of headaches, without fever or unrelated to an underlying morbidity, within the same period of three months. Those who reported having episodes of recurrent headaches were selected for the next phase of the study. The selected students were then administered a structured study instrument incorporating sections comprising of components of the IHS criteria for migraine diagnosis and components of the ID migraine questionnaire, in addition to information on their demographic characteristics [21, 23].

The diagnosis of migraine headache using the ID migraine questionnaire criteria was made following a score of 2 or more, regarded to be attained when a participant with recurrent headaches unrelated to an underlying co-morbidity responded in the affirmative to at least two of the following three component questions:

During the last three months, have you ever had any of the following symptoms concerning your headache pain?

- Did you ever feel nauseous when you had headache pain?

- Did the light trouble you (much more than then when there is no headache)?

- Did your headache ever limit your ability to work, study, or do something you needed to, for at least one day?

We used the IHS criteria as the gold standard for diagnosis of migraine headache which was made when the pattern of recurrent headaches in a participant fulfills the following:

A. At least 5 attacks fulfilling criteria B-D

B. Headache attacks lasting 4-72 hours (untreated or unsuccessfully treated)

C. Headache has at least two of the following characteristics:

- Unilateral location

- Pulsating quality

- Moderate or severe pain intensity

- Aggravation by or causing avoidance of routine physical activity (e.g. walking or climbing stairs) 
D. During headache at least one of the following:

- Nausea and/or vomiting

- Photophobia and phonophobia

Data analysis was conducted with version 20 of the statistical package for social sciences (SPSS). The outcomes obtained with the two diagnostic tools were compared. The ID migraine was compared with the IHS criteria, using the latter as the gold standard, in order to determine the sensitivity, specificity, accuracy and predictive values of the ID migraine, as applicable in the locality. Kappa coefficient was used to determine measures of agreement between the two diagnostic tools. Continuous variables were presented as means and standard deviations, and categorical variables reported as proportions. The level of statistical significance was set at $\mathrm{p}$-value $<0.05$.

\section{RESULTS}

Fifty one of the students comprising 25 (49\%) males and 26 (51\%) females, who reported experiencing recurrent headaches, proceeded to complete the study out of the pool of two hundred and twenty students, constituted by eighty three females and a hundred and thirty seven males. The headache profile, within the previous one year, among the entire pool of students is as shown in Figure-1. The mean age of the participants was 24.22 years \pm 4.575 , with sex specific mean ages of 24.95 years \pm 4.675 and 23.54 years \pm 4.472 for the male and female participants, respectively $(\mathrm{p}=0.301)$. With the use of the International Headache Society (IHS) criteria, $25.5 \%$ of these participants who reported experiencing recurrent headaches were diagnosed to have migraine headaches (see Figure-2), whereas $45.1 \%$ of them were diagnosed to have migraine using the ID migraine tool.

The sensitivity and specificity values we obtained for the ID migraine tool were $69.2 \%$ and $63.1 \%$, respectively. The accuracy, positive predictive and negative predictive values of the ID migraine tool were $64.7 \%, 39.1 \%$ and $85.7 \%$, respectively. There was a minimal level of agreement between the IHS criteria and the ID migraine questionnaire (kappa $=0.258 ; \mathrm{p}=$ 0.043 ). Table-1 shows the comparative outcomes of migraine headache assessment, with the IHS criteria and ID migraine, on the participants with recurrent headaches.

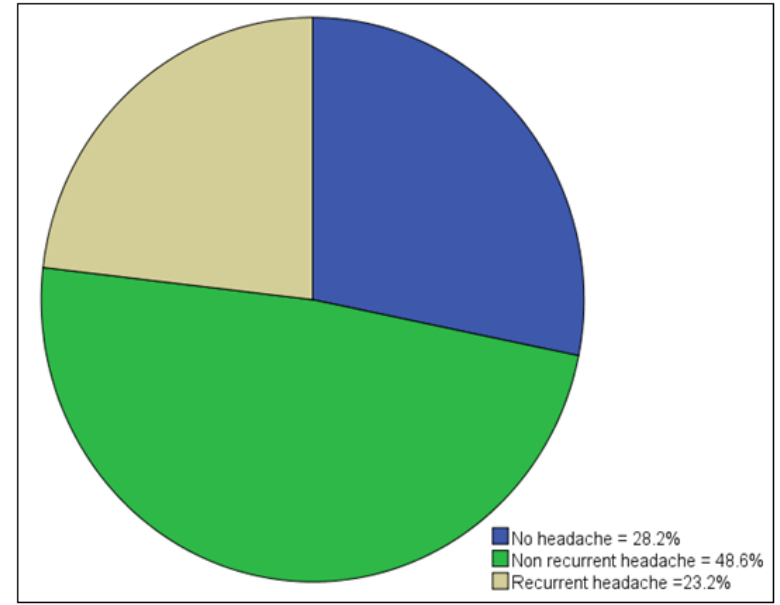

Fig-1: Profile of headaches among the study participants

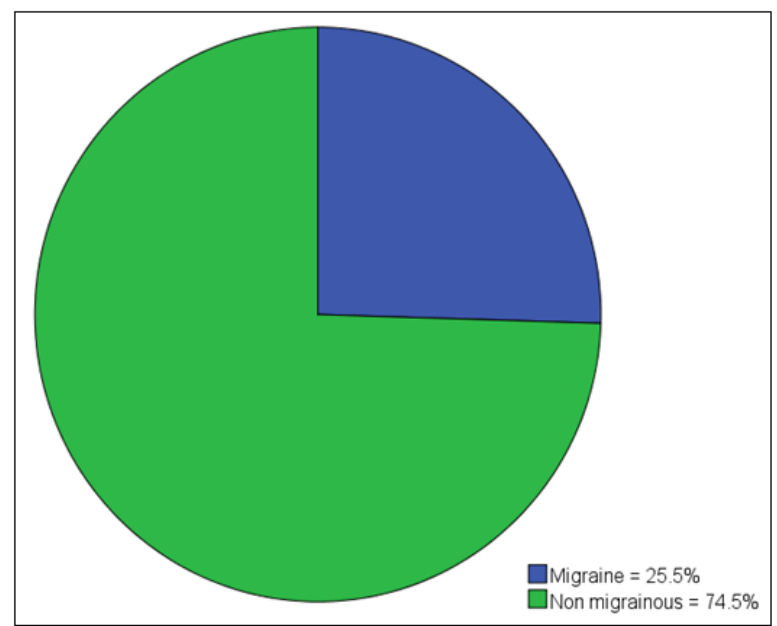

Fig-2: Nature of recurrent headaches among the study participants

Table-1: Outcome of migraine headache assessment with the IHS criteria and ID Migraine

\begin{tabular}{|l|l|l|l|}
\hline & IHS criteria (positive) & IHS criteria (negative) & Total \\
\hline ID migraine (positive) & 9 & 14 & 23 \\
\hline ID Migraine (negative) & 4 & 24 & 28 \\
\hline Total & 13 & 38 & 51 \\
\hline
\end{tabular}

\section{DISCUSSION}

The benefits of reliable rapid screening tools for migraine headache diagnosis towards mitigating the prevalent under-recognition and consequent mistreatment of migraine headache is widely acknowledged [26, 29, 30]. Such benefits are pronounced in resource poor settings like ours, faced with daunting manpower and resource constraints [26, 31, 32].
Our study revealed the ID migraine to have moderate sensitivity and specificity for detecting migraine headache in our locality. Howbeit, its performance in the identification of migraine headache among our study participants, with a sensitivity value of $69.2 \%$, was inferior compared to the sensitivity and positive predictive values of $81 \%$ and $93 \%$, respectively; reported by Lipton et al, who devised the 
ID migraine tool. ${ }^{23}$ Although the trend of less sensitivity value persisted in comparison with other similar studies, the specificity value we obtained for the tool closely mirrors the values reported by researchers in diverse regions of the world; including Latin America, western and eastern Europe [33-36].

The usefulness of the ID migraine tool in our study suffered a setback from the computed positive predictive value which indicates that in the presence of recurrent headaches, the instrument was able to predict the diagnosis of migraine in almost four out of ten cases. Furthermore, we observed a minimal agreement of the ID migraine tool with the IHS criteria used as the gold standard. The difference from other studies could have been influenced by some variations in the study designs, as we employed non probability sampling technique in recruiting the study participants. The latter would largely limit attempts at generalizing the outcome of our study on the wider population. Moreover, our subjects were recruited from seemingly healthy medical students, rather than patients who presented at a hospital as was done in some of these other studies.

The sensitivity value of $69.3 \%$ we obtained for the ID migraine compares favourably with the value of $66.2 \%$ reported in an earlier Nigerian study by Wahab, who used another rapid screening tool known as the 3Question Headache screen, postulated by Cady [22, 37]. Although the sensitivity value from their study is similar to ours, Wahab and his team obtained a better level of performance, with the 3-Question Headache Screen, as the other statistical parameters reported in their study were superior. ${ }^{37}$ Furthermore, their study instrument showed a good agreement with the IHS criteria; suggesting the 3-Question Headache screen to be a better rapid tool for migraine headache diagnosis than the ID migraine, in the primary care setting of our locality.

Our findings indicate that the ID migraine may not be the preferred short item tool for the desired rapid diagnosis of migraine headache in our area. However, the trend of relatively higher negative predictive value and sensitivity, in the presence of low positive predictive value and specificity, we observed in the study suggests that the application of the ID migraine in our locality may be more useful for ruling out, rather than ruling in a diagnosis of migraine headache. The latter suggestion is corroborated by the outcome of a meta-analysis involving thirteen studies. ${ }^{26}$ One could considerably disagree with our submission, on the basis of the challenges associated with our sampling method. It may be of benefit to view our findings from the perspective of a pilot study, necessitating further studies with more robust designs, to further ascertain the usefulness of the ID migraine tool in our setting. Furthermore, in interpreting the implications of our findings, it is worthwhile to take into cognisance that we strictly narrowed the participation in our study to those who reported experiencing recurrent episodes of headaches, not just any form of headache, within the previous three months. It may be pertinent to note that our attempt at the validation of the ID migraine did not consider other subtypes of migraine such as probable or possible migraine, in which one or two features are lacking. Perhaps, incorporating such could positively impact on the usefulness of the ID migraine in our locality. However, such postulates are beyond the scope of our study.

Considering the differences in the performance of the different brief item instruments for migraine headache diagnosis, used in the report by Wahab and in our study, when compared with the IHS criteria as the gold standard, there is need for local comparative studies on the usefulness of the various rapid migraine headache screening tools, the Brief headache screen inclusive, with the purpose of identifying the most suited for our locality [23-25]. Adopting the outcome of such comparative studies would be of huge benefits to resolving the globally acknowledged under-diagnosis and under-treatment of migraine headaches [20]. The benefits would be more pronounced in resource poor settings, as obtained in Nigeria, with unfavourably skewed doctor to patient ratios and the added disadvantage of limited availability of neurologists and other specialist healthcare providers trained in management of headaches $[32,38]$.

\section{CONCLUSION}

In conclusion, our study showed the ID migraine to be of moderate sensitivity and specificity. Its performance in the identification of migraine headache in our locality was less than reported from other parts of the world; especially the western hemisphere. Moreover, the level of agreement with the IHS criteria for migraine headache precludes the ID migraine from being the preferred rapid diagnostic tool for migraine headache in our locality. However, its usefulness in our locality may be more in ruling out, rather than ruling in migraine headache diagnosis in persons who present with recurrent headaches.

\section{ACKNOWLEDGEMENT}

The authors would like to acknowledge the useful contributions and cooperation they received from the class representatives and members of the clinical 1 to 3 classes of the College of Medicine and Health Sciences, University of Calabar, Nigeria.

Conflict of Interests: The authors fully funded the study, and we have no conflict of interests to declare. 


\section{REFERENCES}

1. Bigal, M. E., Liberman, J. N., \& Lipton, R. B. (2006). Age-dependent prevalence and clinical features of migraine. Neurology, 67(2), 246-251.

2. Piovesan, E. J., Kowacs, P. A., Lange, M. C., Pacheco, C., Piovesan, L. D. R. M., \& Werneck, L. C. (2001). Prevalence and semiologic aspects of the idiopathic stabbing headache in a migraine population. Arquivos de neuropsiquiatria, 59(2A), 201-205.

3. Lusic, I. (2001). Population variation in migraine prevalence--the unsolved problem. Collegium antropologicum, 25(2), 695-701.

4. Kowacs, P. A., Piovesan, E. J., Lange, M. C., Werneck, L. C., Tatsui, C. E., Ribas, L. C., ... \& Moreira, A. T. R. (2001). Prevalence and clinical features of migraine in a population of visually impaired subjects in Curitiba, Brazil. Cephalalgia, 21(9), 900-905.

5. Houinato, D., Adoukonou, T., Ntsiba, F., Adjien, C., Avode, D. G., \& Preux, P. M. (2010). Prevalence of migraine in a rural community in south Benin. Cephalalgia, 30(1), 62-67.

6. Vuković, V., Plavec, D., Pavelin, S., Jančuljak, D., Ivanković, M., \& Demarin, V. (2010). Prevalence of migraine, probable migraine and tension-type headache in the Croatian population. Neuroepidemiology, 35(1), 59-65.

7. Lima, A. S., Araújo, R. C. D., Gomes, M. R. D. A., Almeida, L. R. D., Souza, G. F. F. D., Cunha, S. B., \& Pitangui, A. C. R. (2014). Prevalence of headache and its interference in the activities of daily living in female adolescent students. Revista Paulista de Pediatria, 32(2), 256-261.

8. Peterlin, B. L., Gupta, S., Ward, T. N., \& MacGregor, A. (2011). Sex matters: evaluating sex and gender in migraine and headache research. Headache: The Journal of Head and Face Pain, 51(6), 839-842.

9. MacGregor, E. A., Frith, A., Ellis, J., Aspinall, L., \& Hackshaw, A. (2006). Incidence of migraine relative to menstrual cycle phases of rising and falling estrogen. Neurology, 67(12), 2154-2158.

10. World Health Organisation. (2016). Headache disorders. World Health Organisation Factsheets. Geneva; 2016. Available from http://www.who.int/mediacenter/factsheets. (accessed May, 2018).

11. Moskowitz, M. A., \& Macfarlane, R. (1993). Neurovascular and molecular mechanisms in migraine headaches. Cerebrovascular and brain metabolism reviews, 5(3), 159-177.

12. Blau, J. N., \& Dexter, S. L. (1981). The site of pain origin during migraine attacks. Cephalalgia, 1(3), 143-147.

13. Lipton, R. B., Bigal, M. E., Ashina, S., Burstein, R., Silberstein, S., Reed, M. L., ... \& American Migraine Prevalence Prevention Advisory Group.
(2008). Cutaneous allodynia in the migraine population. Annals of neurology, 63(2), 148-158.

14. Noseda, R., \& Burstein, R. (2013). Migraine pathophysiology: anatomy of the trigeminovascular pathway and associated neurological symptoms, cortical spreading depression, sensitization, and modulation of pain. PAIN®, 154, S44-S53.

15. Steiner, T. J., Birbeck, G. L., Jensen, R., Katsarava, Z., Martelletti, P., \& Stovner, L. J. (2010). Lifting The Burden: the first 7 years. Journal Headache Pain, 11, 451-455.

16. Vos, T., Flaxman, A. D., Naghavi, M., Lozano, R., Michaud, C., Ezzati, M., ... \& Abraham, J. (2012). Years lived with disability (YLDs) for 1160 sequelae of 289 diseases and injuries 1990-2010: a systematic analysis for the Global Burden of Disease Study 2010. The lancet, 380(9859), 21632196.

17. World Bank population Estimates and projections. (2012). Available from http://datatopics.worldbank.org/hnp/popestimates\# edu:2012 (accessed July, 2018).

18. Steiner, T. J., Stovner, L. J., \& Vos, T. (2016). Migraine is the third cause of disability worldwide. The Journal of Headache and Pain, 17(1), 104.

19. Steiner, T. J. (2005). Lifting The Burden: the global campaign to reduce the burden of headache worldwide. The journal of headache and pain, 6(5), 373-377.

20. Lipton, R. B., \& Silberstein, S. D. (2001). The role of headache-related disability in migraine management: implications for headache treatment guidelines. Neurology, 56(suppl 1), S35-S42.

21. Headache Classification Committee of the International Headache Society (IHS). (2013). The International Classification of Headache Disorders, 3rd edition (beta version). Cephalalgia, 33(9), 629-808

22. Cady, R. K., Borchert, L. D., Spalding, W., Hart, C. C., \& Sheftell, F. D. (2004). Simple and efficient recognition of migraine with 3 -question headache screen. Headache: The Journal of Head and Face Pain, 44(4), 323-327.

23. Lipton, R. B., Dodick, D., Sadovsky, R. E. A. A., Kolodner, K., Endicott, J., Hettiarachchi, J., \& Harrison, W. (2003). A self-administered screener for migraine in primary care: The ID Migraine ${ }^{\mathrm{TM}}$ validation study. Neurology, 61(3), 375-382.

24. Gervil, M., Ulrich, V., Olesen, J., \& Russell, M. B. (1998). Screening for migraine in the general population: validation of a simple questionnaire. Cephalalgia, 18(6), 342-348.

25. Maizels, M., \& Burchette, R. (2003). Rapid and sensitive paradigm for screening patients with headache in primary care settings. Headache: The Journal of Head and Face Pain, 43(5), 441-450. 
26. Cousins, G., Hijazze, S., Van de Laar, F. A., \& Fahey, T. (2011). Diagnostic accuracy of the ID Migraine: A systematic review and metaanalysis. Headache: The Journal of Head and Face Pain, 51(7), 1140-1148.

27. Anyangwe, S., \& Mtonga, C. (2007). Inequities in the global health workforce: the greatest impediment to health in sub-Saharan Africa. International journal of environmental research and public health, 4(2), 93-100.

28. National Population Commission. (2009). 2006 population census of the Federal Republic of Nigeria, Abuja. Analytical Report at the National level.

29. Lipton, R. B., Scher, A. I., Kolodner, K., Liberman, J., Steiner, T. J., \& Stewart, W. F. (2002). Migraine in the United States: epidemiology and patterns of health care use. Neurology, 58(6), 885-894.

30. Bigal, M. E., Bordini, C. A., \& Speciali, J. G. (2000). Etiology and distribution of headaches in two Brazilian primary care units. Headache: The Journal of Head and Face Pain, 40(3), 241-247.

31. Omideyi, A. K. (2007). Poverty and development in Nigeria: trailing the MDGs?. African Journal of Infectious Diseases, 1(1), 3-17.

32. Adebayo, O., Labiran, A., Emerenini, C. F., \& Omoruyi, L. (2016). Health Workforce for 2016-
2030: Will Nigeria have enough. Inter J Inn Heal Res, 4(1), 9-16.

33. Brighina, F., Salemi, G., Fierro, B., Gasparro, A., Balletta, A., Aloisio, A., ... \& La Naia, F. (2007). A validation study of an Italian version of the "ID Migraine". Headache: The Journal of Head and Face Pain, 47(6), 905-908.

34. Karli, N., Ertas, M., Baykan, B., Uzunkaya, O., Saip, S., Zarifoglu, M., ... \& MIRA study group. (2007). The validation of ID Migraine ${ }^{\mathrm{TM}}$ screener in neurology outpatient clinics in Turkey. The journal of headache and pain, 8(4), 217-223.

35. Gil-Gouveia, R., \& Martins, I. (2010). Validation of the Portuguese version of ID-Migraine. Headache, 50(3), 396-402.

36. de Mattos, A. C. M. T., de Souza, J. A., Filho, P. F. M., Jurno, M. E., \& Velarde, L. G. C. (2017). ID-Migraine $^{\mathrm{TM}}$ questionnaire and accurate diagnosis of migraine (Questionário IDMigraine $^{\mathrm{TM}}$ e o adequado diagnóstico da migrânea). Arq Neuropsiquiatr, 75(7), 446-450.

37. Wahab, K., Ugheoke, A., Okokhere, P., \& Ibekwe T. (2016). Validation of The 3-Question Headache Screen in The Diagnosis of Migraine in Nigeria. Ethiopian Journal Health Sciience, 26(1), 5-8.

38. Owolabi, M. O., Bower, J. H., \& Ogunniyi, A. (2007). Mapping Africa's Way Into Prominence in the Field of Neurology. Arch Neurol, 64(12), 1696-1700. 